\begin{tabular}{|c|c|}
\hline & $\begin{array}{l}\text { International Journal of Trend in Scientific } \\
\text { Research and Development (IJTSRD) }\end{array}$ \\
\hline $\begin{array}{lll}0 & \end{array}$ & International Open Access Journal \\
\hline 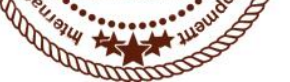 & ISSN No: $2456-6470 \mid$ www.ijtsrd.com | Volume - 2 | Issue -3 \\
\hline
\end{tabular}

\title{
Review: Denoising and Compression Methods
}

\author{
Miss. D. D. Chougule \\ PG Student, Dept. of Electronics Engineering in \\ PVPIT, Budhgaon, Sangli, Maharashtra, India
}

\author{
Prof. K. K. Pandyaji \\ Dept. of Electronics Engineering in PVPIT, \\ Budhgaon, Sangli, Maharashtra, India
}

\begin{abstract}
In this paper, Image recognition is the process of identifying and detecting an object or a feature in a digital image or video. Feature extraction a type of dimensionality reduction that efficiently represents interesting parts of an image as a compact feature vector.
\end{abstract}

A computer revolution has occurred not only in engineering field but also in Medical field, where vast amount of information must be processed very quickly and accurately. High resolution scanning techniques such as digital radiography, tomography, nuclear magnetic resonance, produces an image containing relevant information for medical analysis. The use of computers for handling image data in the healthcare is growing. The amount data produced by modern image generating techniques, / such as Computed Tomography (CT) and Magnetic Resonance (MR), is vast. This huge data might be a problem from a storage point of view or when the data is sent over a network. To overcome such problems data compression techniques are needed.

Keywords: Wavelets, Image Processing, Medical Image, Image De-noising, Image Compression

\section{INTRODUCTION}

The human visual system performs hierarchical edge detection at multiple levels of resolution and wavelet transforms perform a similar analysis. Compression is the process of reducing large data files into smaller files for efficiency of storage and transmission. Data compression techniques are: a. Lossless data compression, b. Lossy data compression. Lossless data compression is nothing but the original data can be reconstructed exactly from compressed data. Lossy data compression in which data after compression and then decompression retrieves a file that is not exactly as the original data as there will be loss of data. Denoising plays a very important role in the field of the medical image pre-processing. [1]

The main purpose of an image-denoising algorithm is to eliminate the unwanted noise level while preserving the important features of an image. In wavelet domain, the noise is uniformly spread throughout the coefficients while mostly the image information is concentrated in the few largest coefficients. [1]

The fidelity of an image after compression is an important aspect of lossy compression methods. The quality of an approach can be evaluated using the difference between the original and the decompressed image. Simultaneous compression and denoising is an important capability of wavelets. These, namely comp-denoisers, are mainly based on thresholding the noise dominated components. The idea is to cancel out the coefficient dominated by noise (in low or high-frequency). This improves the image quality and the compression rate as well. [2]

This work compares the results of different wavelet types. We implemented in the same environment five family of bases: Haar, Daubechies, Biorthogonal, Coiflets and Symlets with many possibilities (it amounts of 36 different variations). They are used to compress a group of natural and synthetic images in different resolutions both with high degradation level $(25=\sigma)$ by Additive White Gaussian Noise (AWGN). The target of the work is to propose a 
comp-denoiser adapted to each type of image and wavelet used.[2]

\section{LITERATURE REVIEW}

Biomedical Image Denoising And Compression In Wavelet Using MATLAB

Vipul Sharan, Naveen Keshari, Tanay Mondal, given various ideas for Biomedical image processing is similar in concept to biomedical signal processing in multiple dimensions. Medical

Images normally have a problem of high level components of noises. Image denoising is an important task in image processing, use of wavelet transform improves the quality of an image and reduces noise level. A novel theory is introduced for analyzing image compression methods that are based on compression of wavelet decompositions. [1]

Influence of the Wavelet Family In The Compression- Denoising Technique On Synthetic And Natural

Aura Conci, Carlos S. Kubrusly, Thomas W. Rauber, In this paper, A denoising procedure based on a thresholding function has been integrated into the JPEG2000 part II standard which is designed to support a wide variety of applications, including the compression and transmission of medical images.

The performance of each approach is evaluated by means of fidelity, comparing the originals without noise versus the same images after compression/denoising and decompression. The goal is to define which combination present the best and the worst results considering the final visual quality. [2]

\section{IMAGE DENOISING COMPRESSION AND \\ VIA WAVELET} THRESHOLDING

Grace Chang, Bin Yu, Martin Vetterli, In this paper, the randomness of the noise. Building on this theme, we explain why compression (via coefficient quantization) is appropriate for filtering noise from signal by making the connection that quantization of transform coefficients approximates the operation of wavelet thresholding for denoising. That is, denoising is mainly due to the zero-zone and that the full precision of the thresholded coefficients is of secondary importance. The method of quantization is facilitated by a criterion similar to Rissanen's minimum description length principle. An important issue is the threshold value of the zero-zone (and of wavelet thresholding). For a natural image, it has been observed that its subband coefficients can be well modeled by a Laplacian distribution.[3]

\section{Adaptive Wavelet Thresholding for Image Denoising and Compression}

S. Grace Chang, Bin Yu, and Martin Vetterli, this paper proposes an adaptive, data-driven threshold for image denoising via wavelet soft-thresholding. The threshold is derived in a Bayesian framework, and the prior used on the wavelet coefficients is the generalized Gaussian distribution (GGD) widely used in image processing applications. The proposed threshold is simple and closed-form, and it is adaptive to each subband because it depends on data-driven estimates of the parameters.

The BayesShrink threshold can aid in the parameter selection of a coder designed with the intention of denoising, and thus achieving simultaneous denoising and compression. Specifically, the zero-zone in the quantization step of compression is analogous to the threshold value in the thresholding function.[4]

\section{FRACTAL-WAVELET IMAGE DENOISING}

M. Ghazel, G. H. Freeman, E.R. Vrscay, In this paper, propose a simple yet effective fractal-wavelet scheme for edge preservingsmoothing of noisy images. Over the past decade, there has been significant interest in fractal coding for the purpose of image compression. Fractal-wavelet transforms were introduced in an effort to reduce the blockiness and computational complexity that are inherent in fractal image compression. Applications of fractal-based coding to other aspects of image processing, however, have received little attention. Recently. the authors proposed a simple yet effective fractal-based image denoising scheme that is applied in the spatial domain of the image. In this paper we extend the application of this fractal denoising scheme to the wavelet domain of the image. We find that when the wavelet transform of the noisy image is simply fractaliy coded, a significant amount of the noise is suppressed.[5]

\section{CHOICE OF WAVELET FILTERS FOR MEDICAL \\ IMAGE COMPRESSION \\ APPROXIMATION \\ AND}

R. S. Dilmaghani, Dr. A. Ahrnadian, N. Maleki, In this paper, wavelet based image coding the choice of wavelets is crucial and determines the coding 
performance. In this paper we investigate effect of applying different types of wavelet Titers belonging to orthogonal and biorthogonal families with different orders on the medical image quality in multiresolution framework. Regularity and linearity of phase response of filters were found to be important factors in chwaing thr proper filter. Our results show that Antonini fiiter $(7 D)$ is the best choice and leads lo more cancelation of aliasing elfeet caused in the analaysis stage and highest peak signal to noise ratio (PSNR) on medical images.[6]

\section{Oriented Wavelet Transform for Image Compression and Denoising}

Vivien Chappelier and Christine Guillemot, In this paper, a new transform for image processing, based on wavelets and the lifting paradigm. The lifting steps of a unidimensional wavelet are applied along a local orientation defined on a quincunx sampling grid. To maximize energy compaction, the orientation minimizing the prediction error is chosen adaptively. A fine-grained multiscale analysis is provided by iterating the decomposition on the low-frequency band. In the context of image compression, the multiresolution orientation map is coded using a quad tree. The rate allocation between the orientation map and wavelet coefficients is jointly optimized in a ratedistortion sense. For image denoising, a Markov model is used to extract the orientations from the noisy image.[7]

\section{Denoising and Image Compression Using Bspline Wavelets}

M.F. Fahmy, G. Abdel Raheem, U. S. Mohamed, Omar F. Fahmy and G. Fahmy, In this paper the results of a recent, a Bspline-based semi-orthogonal wavelet family research on how to construct and implement Bspline ' the wavelet has been constructed. It has been shown that the wavelet basis, is proposed for signal de-noising and image compression.[8]

\section{A Novel Method of Image Steganography in DWT Domain}

Vladimír BÁNOCI, Gabriel BUGÁR, Dušan LEVICKÝ, In this paper, a novel steganographic method for embedding of secret data in still grayscale image. In order to provide large capacity of the secret data while maintaining good visual quality of stegoimage, the embedding process is performed in transform domain of Discrete Wavelet transform (DWT) by modifying of transform coefficients in an appropriate manner.[9]

\section{A Novel Technique for Image Steganography Using Nonlinear Chaotic Map}

S. Thenmozhi, M. Chandrasekaran, This paper presents a novel technique for Image steganography based on DWT, where DWT is used to transform original image (cover image) from spatial domain to frequency domain. The beauty of steganography technology comes out in more pronounced way when there is no absolute relation between stego and original image and it is possible to rebuild the original image in much easier way. As chaotic systems are known to be more random and non-predictable, they can be made utilized in achieving the encryption. The transposition technology of encryption systems requires scrambleness behaviour in order to achieve the encryption of the data.[10]

\section{Image Denoising using Wavelet Transform Method}

Vikas Gupta, Rajesh Mahle, Raviprakash S Shriwas, There have been several numbers of published algorithms and each target to remove noise from original signal. This paper presents a result of some significant work in the area of image denoising it means we explore denoising of images using several thresholding methods such as SureShrink, VisuShrink and BayesShrink. Here we put results of different approaches of wavelet based image denoising methods. To find best method for image denoising is still a valid challenge at the crossing of functional analysis and statistics.[11]

\section{Denoising and wavelet compression of $X$-ray image for teleradiology}

Anita Thakur, Anupama Bhan, Garima Vyas, In this paper, the provision of medical care over long distances by way of videoconferencing and other communication technologies to best consultancy for diagnosis. For teleradiology, image size \& noise improvement is challenging research topic. Mostly Xray images are often corrupted by noise due to a noisy channel or faulty image acquisition device. In median filter methods are used for denoising and wavelet based compression is implementated for transmission. The wavelet based method used in this paper is Set Partitioning in Hierarchical Trees Algorithm (SPIHT) which is a type of Multiwavelet transforms. The image quality of the system has been evaluated based on compression ratio, peak signal to noise ratio and entropy.[12] 


\section{CONCLUSION}

In this paper we have taken a biomedical image for de-noising and compression in Wavelet Toolbox specially Wavelet $2 \mathrm{D}$ in MATLAB and MATLAB command prompt using step by step. As a result we get the compressed image as well as noise free in vertical, horizontal and diagonal details and got energy ratio. After doing this there is almost no detectable deterioration in the image quality.

A quality performance analysis was done using the Signal to Noise Ratio (SNR) and Peak Signal to Noise Ratio (PSNR) as evaluation criteria and subtraction the original noiseless version of each image from the reconstructed image after the thresholding process. All tests have been analysed and combined in different manners.

\section{REFERENCES}

1. Vipul Sharan, Naveen Keshari, Tanay Mondal," Biomedical Image Denoising and Compression in Wavelet using MATLAB", International Journal of Innovative Science and Modern Engineering (IJISME) ISSN: 2319-6386, Volume-2, Issue-6, May 2014.

2. Aura Conci, Carlos S. Kubrusly, Thomas W. Rauber, "Influence of the Wavelet Family In The Compression- Denoising Technique On Synthetic And Natural , "IEEE, 978-1-4244-4530-1,2009.

3. Grace Chang, Bin $\mathrm{Yu}$, Martin Vetterli "Image Denoising Via Lossy Compression And Wavelet Thresholding", Ieee Page No- 8186-81837/97,1997.

4. S. Grace Chang, Bin Yu, And Martin Vetterli,"Adaptive Wavelet Thresholding For Image Denoising And Compression", Ieee Transactions On Image Processing, Vol. 9, No. 9, September 2000.

5. M. Ghazel, G. H. Freeman, E.R. Vrscay," FractalWavelet Image Denoising", Ieee, Page No-78037622, Vol. No-6,2002.

6. R. S. Dilmaghani, Dr. A. Ahrnadian, N. Maleki, “CHOICE OF WAVELET FILTERS FOR MEDICAL IMAGE COMPRESSION AND APPROXIMATION", IEEE, page no-7803-7612 vol.no-9, oct, 2002.

7. Vivien Chappelier And Christine Guillemot,"Oriented Wavelet Transform For
Image Compression And Denoising", Ieee Transactions On Image Processing, Vol. 15, No. 10, October 2006.

8. M.F. Fahmy, G. Abdel Raheem, U. S. Mohamed, Omar F. Fahmy And G. Fahmy,"Denoising And Image Compression Using Bspline Wavelets", IEEE,Page No-978-1005, Vol.No-5,2008.

9. Vladimír BÁNOCI, Gabriel BUGÁR, Dušan LEVICKÝ," A Novel Method Of Image Steganography In DWT Domain" IEEE,Page No978,Vol.No-7,2011.

10. S. Thenmozhi， M. Chandrasekaran,"A Novel Technique For Image Steganography Using Nonlinear Chaotic Map" Ieee ,Page No-46734603, Vol.No-12,2012.

11. Vikas Gupta, Rajesh Mahle, Raviprakash S Shriwas," Image Denoising Using Wavelet Transform Method "IEEE, Page No-46735999, Vol No-3,2013.

12. Anita Thakur, Anupama Bhan, Garima Vyas,"Denoising And Wavelet Compression Of X-Ray Image For Teleradiology" IEEE ,Page No4799-6896, Vol No-1,2014. 